\title{
Safety of Inguinal Hernia Repair in the Elderly with Perioperative Continuation of Antithrombotic Therapy
}

\author{
Gonish Hada $^{\mathrm{a}}$ Sen Zhang ${ }^{\mathrm{a}}$ Yinghan Song $^{\mathrm{b}}$ Mukesh Jaiswar ${ }^{\mathrm{a}}$ Yanyan Xie $^{\mathrm{a}}$ \\ Fushan Jian $^{a}$ Wenzhang Lei ${ }^{\mathrm{a}}$ \\ ${ }^{a}$ Department of Gastrointestinal Surgery, Hernia Center, West China Hospital, Sichuan University, Chengdu, China; \\ ${ }^{b}$ Department of Day Care Surgery, West China Hospital, Sichuan University, Chengdu, China
}

\section{Keywords}

Inguinal hernia - Antithrombotic therapy · Bleeding •

Thromboembolism · Elderly

\begin{abstract}
Introduction: This study aimed to evaluate the safety of an inguinal hernia repair (IHR) under local anesthesia (LA) in the elderly with a perioperative continuation of antithrombotic therapy (AT). Methods: A total of 120 patients undergoing elective primary IHR between August 2018 and August 2019 at the West China Hospital of China were prospectively studied, among which 60 patients also had coexisting cardiovascular diseases and had a continuation of AT perioperatively (antithrombotic group); the other 60 patients were not on any prior AT (control group). The primary endpoints were intra- and postoperative hemorrhagic complications, the required interventions for complications based on the ClavienDindo classification, and postoperative thromboembolic complications. The secondary endpoints were nonhemorrhagic complications, intraoperative duration, and postoperative length of stay (LOS). Results: None of the patients in both groups had significant intraoperative bleeding $>10 \mathrm{~mL}$, and there were no significant differences between the 2 groups in terms of the postoperative hemorrhagic complications: bruising ( 2 vs. $0 \%, p=1.000$ ), serosanguinous soakage (7 vs. $3 \%, p=0.679$ ), and no hematoma was observed. Interventions required for encountered complications based on the Clavien-Dindo classification grade I ( 7 vs. $5 \%, p=1.000$ ) were assessed. There were no episodes of postoperative
\end{abstract}

thromboembolic complications within 60 days in both groups. There were also no significant differences between the 2 groups in terms of nonhemorrhagic complications, intraoperative duration, and postoperative LOS ( $p>0.05$ in all). Conclusions: The perioperative continuation of AT did not increase the risk of intra- and postoperative hemorrhagic complications following IHR in the elderly. Thus, IHR under LA seems to be safe and feasible in this setting.

(C) 2020 S. Karger AG, Base

\section{Introduction}

Inguinal hernia has been a key presence in the elderly, especially in the male population [1]. The tension-free hernia repair under local anesthesia (LA) has now become a commonly accepted method for inguinal hernia repair (IHR), especially in the elderly. There is a high incidence of cardiovascular comorbidities in this subgroup of the population [2]. As a result, a large portion of this population needing surgery is on chronic antithrombotic therapy (AT). Despite their high efficiency for primary and secondary prophylaxis of cardiovascular disease, these drugs are always associated with inevitable bleeding risks and complications. Hemorrhagic adverse effects are more distinct in the elderly than in adults. These findings may be related to low body weight, decreased hepatic and renal metabolism, multiple coexisting comorbidities, and polypharmacy with an adverse drug-to-drug interaction in these subgroups $[3,4]$. 
The transient perioperative interruption of these drug therapies has been found to increase the adverse risks of thromboembolic events. There are reports of numerous incidences of thromboembolic events, such as stroke, myocardial infarction (MI), stent occlusion, thrombosis, and even death, after the perioperative withdrawal of AT drugs [5, 6]. Again, the risk for thromboembolic events is found to be more apparent in the elderly; this may be related to suboptimal platelet inhibition and high residual platelet reactivity during therapy in these subgroups [7].

The clinical decision-making about the perioperative reversal of anticoagulation or cessation of antiplatelets in these subgroups is, therefore, quite challenging. It is very crucial to analyze and balance the perioperative bleeding risk with the thromboembolic risk during the optimization of AT in these subgroups. The few recognized guidelines have recommended the temporary discontinuation of AT with or without perioperative bridging therapy depending on individual thromboembolic risk [8-10]. However, in recent decades, the routine practice of withholding these drugs for the preparation of low-bleeding-risk surgical or diagnostic interventions is being questioned. Multiple research has reported the safety of dental, ophthalmologic, and dermatologic surgeries as well as invasive endoscopic interventions with a continuation of AT perioperatively $[11,12]$. In a similar context, both the American Academy of Neurology (AAN) and the American College of Chest Physicians (ACCP) have also recommended continuing AT perioperatively for such low-bleeding-risk surgeries $[11,12]$. However, the safety of IHR especially in the elderly with perioperative AT has to be analyzed.

In this prospective study, we mainly focus on the safety and feasibility of IHR under LA in the elderly male population with perioperative continuation of AT. We compared their intra- and postoperative hemorrhagic and nonhemorrhagic complications with the control group.

\section{Methods}

\section{Patients}

In this prospective cohort study, a total of 120 elderly male patients admitted for an elective primary IHR at the Gastrointestinal Department of West China Hospital of China from August 2018 to April 2019 were enrolled, out of which 60 patients also had coexisting cardiovascular diseases for which they were on chronic AT (namely aspirin, clopidogrel, and warfarin) and were asked to continue a full dose of AT perioperatively (antithrombotic group). Sixty other relatively healthy patients comparable for age and gender who had not been on any prior AT were also enrolled (control group). The inclusion criteria were elderly patients defined by age $>60$ years, male gender, and primary inguinal hernia (unilateral or bilateral), whereas exclusion criteria included a strangulated, incarcerated, and recurrent hernia. Patients with a known history of primary and secondary bleeding disorders were also excluded from the study. The reporting was done according to the STROBE statement checklist for cohort studies.

\section{Surgical Techniques}

All surgeries were performed by the same team led by a single experienced hernia surgeon. Local anesthetic solutions were composed of $20 \mathrm{~mL}$ of $2 \%$ lidocaine, $10 \mathrm{~mL}$ of $10 \%$ ropivacaine mixed

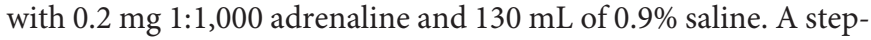
wise local infiltration of anesthetic solutions was performed throughout the surgery. The hernia defect was repaired with tension-free implantation of a double-layer polypropylene mesh (UltraPro Hernia System [UHS] mesh, Ethicon, Norderstedt, Germany) by the standard UHS. A meticulous blunt dissection of the hernia sac along tissue planes, avoiding excessive sharp dissection deep into the pre-peritoneal space and injuries to deep epigastric vessels, and avoiding the excessive resection of the distal hernia sac, was done to minimize the risk of hemorrhagic complications. Bleeding witnessed during the procedure was controlled using monopolar cautery or by ligation with fine suture. No drainage tube was used. Postoperative bedside follow-up was carried out on $12 \mathrm{~h}$ and day 1 and post-discharge telephonic follow-up on day 3 and 7 for hemorrhagic complications and day 60 for thromboembolic complications.

\section{Outcome Parameters}

The following outcome parameters were prospectively collected: demographic data including age, gender, body mass index, comorbidities, American Society of Anesthesiologists (ASA) score, lab reports, and data related to antithrombotic medications.

Primary endpoints were defined as follows:

- Bleeding complications (intraoperative bleeding, bruising, hematoma, and serosanguinous soakage from wound dressing). Intraoperative bleeding $>10 \mathrm{~mL}$ was only considered significant for statistical analysis.

- Postoperative bleeding events requiring additional intervention based on the Clavien-Dindo classification. The ClavienDindo classification grade I includes administration of antipyretics, analgesics, antiemetics, diuretics, and physiotherapy. It also includes minor bedside interventions like administration of intravenous fluids, urinary catheterization, nasogastric decompression, and wound exploration and drainage [13].

- Postoperative thromboembolic events within 60 days.

The secondary endpoints were intraoperative parameters, postoperative length of stay, nonhemorrhagic complications (seroma, inguinoscrotal swelling, wound infection), need for blood transfusion, or reoperation or readmission, and hernia recurrence.

\section{Statistical Methods}

All the statistical analyses were done using SPSS statistics version 23.0.0 for MAC.OSX. Continuous variables were presented as mean value (SD) or median (interquartile range) after testing the normality distribution of variables, whereas categorical variables were presented as number (\%). The Student $t$ test, one-way ANOVA test, or Mann-Whitney U test was used for continuous variables, and categorical variables were compared with the $\chi^{2}$ test or Fisher's exact test. All comparisons were two-tailed, and the difference was statistically significant with a $p$ value $<0.05$.

\section{Results}

The demographic data for both groups are shown in Table 1. Both groups had comparable age, gender, body mass index, and duration of hernia symptoms $(p>0.05$ for all). Their ages ranged from 60 to 89 years with a median of 73 years. All patients involved in this study were male 
Table 1. Patients' demographic data $(n=120)$

\begin{tabular}{|c|c|c|c|}
\hline Variables & $\begin{array}{l}\text { Antithrombotic } \\
\text { group }(n=60)\end{array}$ & $\begin{array}{l}\text { Control } \\
\text { group }(n=60)\end{array}$ & $p$ value \\
\hline Age, years ${ }^{\mathrm{a}}$ & $72.77(7.02)$ & $72.33(7.61)$ & 0.746 \\
\hline Male gender & $60 / 60(100)$ & $60 / 60(100)$ & $\mathrm{N} / \mathrm{A}$ \\
\hline $\mathrm{BMI}, \mathrm{kg} / \mathrm{m}^{2 \mathrm{a}}$ & $22.72(2.80)$ & $22.93(2.42)$ & 0.661 \\
\hline \multicolumn{4}{|l|}{ Associated comorbidities } \\
\hline Cardiac disease & $54(90)$ & $6(10)$ & $<0.001^{*}$ \\
\hline CAD & $40(67)$ & - & N/A \\
\hline Valvular heart disease & $9(15)$ & - & N/A \\
\hline Atrial fibrillation & $5(8)$ & - & N/A \\
\hline Prior stroke/TIA & $6(10)$ & - & N/A \\
\hline HTN & $29(48)$ & $12(20)$ & $0.001^{*}$ \\
\hline $\mathrm{DM}$ & $12(20)$ & $4(7)$ & $0.032^{*}$ \\
\hline COPD & $4(7)$ & $5(8)$ & 1.000 \\
\hline $\mathrm{BPH}$ & $6(10)$ & $8(13)$ & 0.569 \\
\hline Duration of symptoms, years ${ }^{b}$ & $1.00(1.54)$ & $0.83(0.75)$ & 0.236 \\
\hline Perioperative INR & $1.21(0.48)^{\mathrm{c}}$ & $0.97(0.08)$ & $<0.001^{*}$ \\
\hline Perioperative platelets, $10^{9} / \mathrm{L}^{\mathrm{b}}$ & $147.5(52)$ & $149.5(38)$ & 0.713 \\
\hline \multicolumn{4}{|l|}{ ASA score } \\
\hline 1 & $0(0)$ & $6(12)$ & $\mathrm{N} / \mathrm{A}$ \\
\hline 2 & $21(36)$ & $37(62)$ & $0.004^{*}$ \\
\hline 3 & $39(64)$ & $17(26)$ & $<0.001^{*}$ \\
\hline
\end{tabular}

BMI, body mass index; CAD, coronary artery disease; TIA, transient ischemic attack; HTN, hypertension; $\mathrm{DM}$, diabetes mellitus; COPD, chronic obstructive pulmonary disease; $\mathrm{BPH}$, benign prostatic hyperplasia; INR, international normalized ratio; ASA, American Society of Anesthesiologists; N/A, not assessed. Continuous variables are presented as ${ }^{\mathrm{a}}$ mean (SD) or ${ }^{\mathrm{b}}$ median (IQR), whereas categorical variables are presented as $n(\%) .{ }^{\mathrm{c}}$ Mean INR of including both anticoagulants and antiplatelets. ${ }^{*} p<0.05$.

patients. In the antithrombotic group, we found a higher prevalence of cardiovascular comorbidities ( 93 vs. $6 \%, p<$ 0.001 ), hypertension ( 48 vs. $20 \%, p=0.001$ ), diabetes mellitus ( 20 vs. $7 \%, p=0.032$ ), and ASA grading $>2$ (64 vs. $26 \%$, $p<0.001)$. The data related to AT in the antithrombotic group are shown in Table 2. Of those who had continuation of AT, $33(55 \%)$ patients were on aspirin, 8 (13\%) patients on clopidogrel, 4 (7\%) patients on DAPT with aspirin and clopidogrel, and the remaining 15 (25\%) patients were on warfarin anticoagulation therapy. The median duration of AT was 2 years. The preoperative international normalized ratio (INR) value of patients on anticoagulants was $1.97(0.41)$ and $0.96(0.06)$ for patients on antiplatelets.

As shown in Table 3, there was no statistically significant difference between both groups in terms of the intraoperative duration for unilateral repair (29.4 vs. $29.1 \mathrm{~min}$, $p=0.577$ ) and for bilateral repair (45.0 vs. $43.9 \mathrm{~min}, p=$ 0.420 ), where 8 patients from each group had a bilateral hernia repair. None of our patients had significant intraoperative bleeding of $>10 \mathrm{~mL}$ in any of the groups. No difference was observed in terms of postoperative length of stay $(p=0.628)$. Regarding the incidence of postoperative hemorrhagic complications, as shown in Table 4, no statistical difference was found between both groups: bruising ( 1 vs. 0 cases, $p=1.000$ ), serosanguinous soakage
Table 2. Data related to antithrombotic drugs in the AT group $(n=60)$

\begin{tabular}{lc}
\hline Variables & $\begin{array}{l}\text { Antithrombotic } \\
\text { group }(n=60)\end{array}$ \\
\hline Antiplatelets & \\
$\quad$ Aspirin & $33(55)$ \\
Clopidogrel & $8(13)$ \\
DAPT & $4(7)$ \\
Warfarin & $15(25)$ \\
Preoperative INR & \\
$\quad$ Antiplatelet & $0.96(0.06)$ \\
$\quad$ Anticoagulant & $1.97(0.41)$ \\
Duration of therapy, years ${ }^{b}$ & $2.00(4.00)$ \\
Indications & \\
$\quad$ Coronary artery disease & $24(40)$ \\
$\quad$ PCI with stent implantation & $16(27)$ \\
Atrial fibrillation & $5(8)$ \\
Prosthetic heart valve replacement & $9(15)$ \\
$\quad$ Prior stroke & $6(10)$ \\
\hline
\end{tabular}

DAPT, Dual antiplatelet therapy; INR, international normalized ratio; PCI, percutaneous coronary intervention. Continuous variables are presented as ${ }^{\mathrm{a}}$ mean (SD) or ${ }^{\mathrm{b}}$ median (IQR), whereas categorical variables are presented as $n(\%)$. 
Table 3. Intraoperative parameters

\begin{tabular}{|c|c|c|c|}
\hline Variables & $\begin{array}{l}\text { Antithrom- } \\
\text { botic group } \\
(n=60)\end{array}$ & $\begin{array}{l}\text { Control } \\
\text { group } \\
(n=60)\end{array}$ & $p$ value \\
\hline \multicolumn{4}{|l|}{ Operation time, $\min ^{\mathrm{a}}$} \\
\hline Unilateral repair & $29.4(3.52)$ & $29.1(3.49)$ & 0.577 \\
\hline Bilateral repair & $45.0(3.02)$ & $43.9(2.36)$ & 0.420 \\
\hline \multicolumn{4}{|l|}{ Intraoperative bleeding loss } \\
\hline$<10 \mathrm{~mL}$ & $60(100)$ & $60(100)$ & 1.000 \\
\hline$>10 \mathrm{~mL}$ & - & - & $\mathrm{N} / \mathrm{A}$ \\
\hline \multicolumn{4}{|l|}{ Hernia laterality } \\
\hline Right & $25(42)$ & $28(47)$ & 0.304 \\
\hline Left & $27(45)$ & $24(40)$ & 0.306 \\
\hline Bilateral & $8(13)$ & $8(13)$ & 1.000 \\
\hline \multicolumn{4}{|l|}{ Types } \\
\hline Direct & $23(38)$ & $20(33)$ & 0.568 \\
\hline Indirect & $34(57)$ & $36(60)$ & 0.711 \\
\hline Pantaloon & $3(5)$ & $4(7)$ & 1.000 \\
\hline \multicolumn{4}{|l|}{ Size of hernia defect } \\
\hline$<1.5 \mathrm{~cm}$ & $6(10)$ & $9(15)$ & 0.408 \\
\hline $1.5-3.0 \mathrm{~cm}$ & $41(68)$ & $40(67)$ & 0.846 \\
\hline$>3.0 \mathrm{~cm}$ & $13(22)$ & $11(18)$ & 0.648 \\
\hline \multicolumn{4}{|l|}{ Content of hernia sac } \\
\hline None & $39(65)$ & $38(63)$ & 0.849 \\
\hline Omentum & $12(20)$ & $16(27)$ & 0.388 \\
\hline Small bowel & $9(15)$ & $6(10)$ & 0.408 \\
\hline Others ${ }^{\mathrm{b}}$ & - & - & $\mathrm{N} / \mathrm{A}$ \\
\hline \multicolumn{4}{|l|}{ Protrusion of hernia sac } \\
\hline Limited to inguinal canal & $46(77)$ & $53(88)$ & 0.926 \\
\hline Into scrotum & $14(23)$ & $7(12)$ & 0.926 \\
\hline \multicolumn{4}{|l|}{ Injuries to epigastric vessels } \\
\hline No injury & $58(97)$ & $57(95)$ & 0.648 \\
\hline Injury and ligated & $2(3)$ & $3(5)$ & 1.000 \\
\hline Injuries to spermatic vessels & $3(5)$ & $1(2)$ & 0.619 \\
\hline Other visceral injuries ${ }^{\mathrm{c}}$ & - & - & $\mathrm{N} / \mathrm{A}$ \\
\hline \multicolumn{4}{|l|}{ Hernia sac reduction } \\
\hline Complete reduction & $37(62)$ & $31(52)$ & 0.269 \\
\hline High ligation of hernia sac & $23(38)$ & $29(48)$ & 0.269 \\
\hline
\end{tabular}

N/A, not assessed. Continuous variables are presented as a mean (SD), whereas categorical variables are presented as $n(\%)$.

${ }^{\mathrm{b}}$ Includes uncommon contents like appendix, ovary, urinary bladder, and large bowel. ${ }^{c}$ Includes injury to bowel, major vessels, and vas deferens.

( 4 vs. 2 cases, $p=0.679$ ), and no cases of hematoma were observed. There was also no significant difference between both groups in terms of the predetermined secondary endpoints. The follow-up complications, such as wound seroma ( 1 vs. 1 case, $p=1.000$ ), delayed wound healing ( 2 vs. 1 case, $p=1.000$ ), inguinoscrotal swelling ( 4 vs. 2 cases, $p=0.679$ ), and urinary retention ( 0 vs. 1 case, $p=1.000$ ), were recorded. No episodes of postoperative thromboembolic complications, wound infection, recurrence of the hernia, and the need for mesh removal were observed in any of the groups.

Regarding the need for intervention for encountered postoperative complications, there was also no statisti-
Table 4. Postoperative outcomes

\begin{tabular}{|c|c|c|c|}
\hline Endpoints & $\begin{array}{l}\text { Anti- } \\
\text { throm- } \\
\text { botic } \\
(n=60)\end{array}$ & $\begin{array}{l}\text { Control } \\
(n=60)\end{array}$ & $p$ value \\
\hline Mean postoperative LOS, days & 1.40 & 1.18 & 0.430 \\
\hline \multicolumn{4}{|l|}{ Hemorrhagic complications } \\
\hline Bruising & $1(2)$ & - & 1.000 \\
\hline Hematoma & - & - & $\mathrm{N} / \mathrm{A}$ \\
\hline $\begin{array}{l}\text { Serosanguinous soakage on } \\
\text { dressing }\end{array}$ & $4(7)$ & $2(3)$ & 0.679 \\
\hline \multicolumn{4}{|l|}{ Nonhemorrhagic complications } \\
\hline Wound seroma & $1(2)$ & $1(2)$ & 1.000 \\
\hline Delayed wound healing & $2(3)$ & $1(2)$ & 1.000 \\
\hline Wound infection & - & - & $\mathrm{N} / \mathrm{A}$ \\
\hline Inguinoscrotal swelling & $4(7)$ & $2(3)$ & 0.679 \\
\hline Urinary retention & - & $1(2)$ & 1.000 \\
\hline Thromboembolic events in 60 days & - & - & $\mathrm{N} / \mathrm{A}$ \\
\hline Recurrence & - & - & $\mathrm{N} / \mathrm{A}$ \\
\hline Death & - & - & $\mathrm{N} / \mathrm{A}$ \\
\hline \multicolumn{4}{|l|}{ Interventions } \\
\hline Clavien-Dindo classification grade I & $4(7)$ & $3(5)$ & 1.000 \\
\hline Need for BT & - & - & $\mathrm{N} / \mathrm{A}$ \\
\hline Reoperation & - & - & N/A \\
\hline Readmission & - & - & $\mathrm{N} / \mathrm{A}$ \\
\hline
\end{tabular}

Values are (\%) except for LOS. N/A, not assessed; LOS, length of stay; BT, blood transfusion.

cally significant difference between both groups: ClavienDindo classification grade I ( 4 vs. 3 cases, $p=1.000$ ) was observed. All bruising and seromas were treated within a couple of weeks with conservative treatments, such as warm compression and bed rest. No patient required blood transfusion, reoperation, or readmission. A case of urinary retention observed in the control group resolved spontaneously after a few episodes of intermittent urinary catheterization. Out of 6 cases ( 4 vs. 2 cases, respectively) of inguinoscrotal swelling observed, only 3 cases ( 2 vs. 1 case, respectively) needed intervention. Anti-inflammatory and analgesic drugs were prescribed, and $\mathrm{T}$ pressure bandage was applied. A total of 3 cases of delayed wound healing were observed ( 2 vs. 1 case, respectively). Bedside wound exploration and packing were done. It healed after a series of regular dressing. No antibiotics were administered.

In Table 5, we compared the intra- and postoperative hemorrhagic complications among the patients in the AT group based on different types of antithrombotic drugs used. None of the patients had intraoperative bleeding $>10 \mathrm{~mL}$. In terms of postoperative hemorrhagic complications, only 1 case (2\%) of postoperative bruising was observed in a clopidogrel user, and 2 cases (3\%) on aspirin and 1 case $(2 \%)$ each on clopidogrel and warfarin had serosanguinous soakage $(p=0.872)$. A total of 3 cases 
Table 5. Endpoints in the antithrombotic group $(n=60)$

\begin{tabular}{llllll}
\hline Variables & Aspirin & Clopidogrel & DAPT & Warfarin & $p$ value \\
\hline Patients & $33(55 \%)$ & $8(13 \%)$ & $4(7 \%)$ & $15(25 \%)$ & \\
Hemorrhagic complications & - & $1(2)$ & - & - & 0.086 \\
$\quad$ Bruising & - & - & - & - & N/A \\
$\quad$ Hematoma & $2(3)$ & $1(2)$ & - & $1(2)$ & 0.872 \\
$\quad$ Serosanguinous soakage & $1(2)$ & - & - & - & 0.852 \\
Nonhemorrhagic complications & $2(3)$ & - & - & - & 0.656 \\
$\quad$ Wound seroma & - & $1(2)$ & $1(2)$ & $2(3)$ & 0.113 \\
$\quad \begin{array}{l}\text { Delayed wound healing } \\
\text { Inguinoscrotal swelling }\end{array}$ & $5(8)$ & $3(5)$ & $1(2)$ & $3(5)$ & 0.539 \\
$\quad \begin{array}{l}\text { Total complications } \\
\text { Interventions }\end{array} \quad$ Clavien-Dindo classification grade I & $3(6)$ & - & - & $1(2)$ & 0.774 \\
\hline
\end{tabular}

Values are $n$ (\%). DAPT, dual antiplatelet therapy; N/A, not assessed.

$(6 \%)$ on aspirin and 1 case $(2 \%)$ on warfarin required intervention ( $p=0.78)$ for encountered complications (Clavien-Dindo classification grade I).

\section{Discussion}

An inguinal hernia is a common disease in the geriatric population [14]. Given the increased aging of our population, along with recent advances in the medical treatment of chronic cardiovascular diseases, a high percentage of elderly patients needing IHR surgery are on chronic AT [15]. The transient perioperative interruption of AT increases the risk of thromboembolic complications, while the perioperative continuation increases the risk of hemorrhagic surgical complications. As advanced age is already associated with an increased risk of ischemic events and thrombosis, the perioperative withdrawal of AT will further escalate the risk of thromboembolic events. A study reported age older than 65 years as a risk factor for developing postoperative complications in IHR [16].

According to hernia registration studies, patients receiving AT or patients with existing coagulation disorders reported a 4 -fold increase in the risk of subsequent bleeding after surgery [17]. The American College of Surgeons (ACS) guidelines have recommended the withdrawal of warfarin for at least 6 days before low-bleedingrisk procedures with or without bridging therapy varying according to individual thromboembolic risk [8], whereas in the case of patients on antiplatelet therapy, both the ACS and the American College of Cardiology (ACC) guidelines have recommended continuing aspirin and discontinuing P2Y12 receptor inhibitor for preparation of low-bleeding-risk surgery $[8,9]$. However, surgeons often underestimate the adverse risk of ischemic events related to the perioperative withdrawal of AT or low-mo- lecular-weight heparin bridging therapy. Relapse or recurrence of stroke, deep vein thrombosis, and MI is not uncommon in these subgroups $[18,19]$. A higher incidence of ischemic stroke was found to have occurred within 60 days of anticoagulant withdrawal with a peak occurring at 5-6 days $[18,20]$.

Withdrawal of aspirin in coronary artery disease patients was found to increase the risk of MI by 4 times in comparison to controls [21]. These findings could be related to abnormally elevated platelet reactivity and increased thromboxane A2 activity after withdrawal [22]. The incidence of major ischemic events peaked on days 3-9 of aspirin withdrawal, with events occurring even earlier with clopidogrel [23]. Besides, surgical trauma itself is known to create a hypercoagulable period postoperatively (increased platelet activation/aggregation and decreased fibrinolysis) increasing further risk for thromboembolic complications [24].

In our study, none of our patients had significant intraoperative bleeding of $>10 \mathrm{~mL}$. Only a few events of minor complications were observed, which were managed with relatively simple conservative measures. The majority of observed complications were nonhemorrhagic complications in both groups. The outcomes were satisfactory even for bilateral hernia repairs. Our results are in accordance with earlier studies conducted in inguinal hernia patients undergoing open Lichtenstein repair in a similar setting $[25,26]$. However, no study was known to compare the outcomes of IHR using the UHS technique under LA. A study reported preoperative warfarin usage (INR $>3$ ) as a significant variable for inguinal hematoma following IHR [27]. In a similar context, the British Society of Gastroenterology (BSG) and European Society of Gastrointestinal Endoscopy (ESGE) guidelines for high-risk endoscopic procedures have also recommended to check and ensure preoperative INR $<1.5$ after 
discontinuing warfarin for 5 days prior to procedures [10]. However, in the current study, we found that intraand postoperative bleeding complications were minimal even with a preoperative INR value of 1.97 (0.41) among the warfarin users.

With coexisting cardiovascular and respiratory disease in the elderly, LA has several advantages over regional or general anesthesia. Fewer complications, no postoperative sedation or drowsiness, minimal physiological disturbances, early ambulation and bowel motions, shorter hospital stay, and low recurrence rate are its advantages [28]. While the addition of adrenaline to the anesthetic solution not only improves its onset and half-life, it also diminishes regional blood flow, thus reducing bleeding. The administration of an anesthetic solution also provides a comparatively bloodless surgical dissection plane during dissection of the hernia sac from other contents in the inguinal canal. However, an elderly population, particularly those affected by hypertension, severe cardiovascular disease (i.e., recent MI, unstable angina, decompensated heart failure, arrhythmias, and severe valvular disease), is particularly vulnerable to the adverse effect of adrenaline even at the recommended therapeutic doses $(1: 200,000)$ [29]. Therefore, a relatively lower concentration of adrenaline was used in the current study.

Our study provides strong evidence to support the perioperative continuation of AT in IHR under LA, especially in high-risk elderly where the thromboembolic risk often outweighs the hemorrhagic risk associated with surgical interventions. Thus, patients no longer need to wait for the complete reversal of the AT effect nor do they need inefficacious perioperative bridging therapy. This will decrease the potential risk for thromboembolic events and will also decrease the overall length of stay and the hospitalization expenses of patients. In one study, the average length of hospital stay was 4.31 versus 1.61 days in the patients who discontinued aspirin in comparison to those who continued [25]. Furthermore, without this preoperative delay, the theoretical risk for strangulation and incarceration of the hernia is also reduced, as the reported risk of morbidity and mortality of patients following emergency surgery for strangulation and incarceration of a hernia is high [30].

However, we do not recommend that all hernia surgeries should be performed with AT continuation in all patients. The ideal decision should still be made by proper individual assessment of the benefits versus risks and the surgeon's operative skills. We suggest postponing the surgery in patients with an active bleeding history and INR $>3$ until proper optimization has been done. Although IHR is generally considered as low-bleeding-risk surgery, each patient's individual bleeding risk must be assessed with validated scoring systems, such as HAS-BLED,
HEMORR ${ }_{2}$ HAGES scores, etc. A detailed history should be taken focusing on the patient characteristics that increase bleeding risk, such as hypertension, stroke, bleeding history, deranged liver and kidney function, drugs, alcohol consumption, and labile INR value. The history related to bleeding complications in previous surgeries or trauma is also helpful for reference. The surgeon's clinical experience with hernia surgery is the key determinant. A good anatomical knowledge, meticulous dissection, and adequate hemostasis during surgery are still reasonable. Early and careful postoperative assessment for potential hemorrhagic complications, such as hematoma, bruising, etc., should be conducted particularly in patients with large hernia defects and sac.

The current study may have some limitations. The study population was limited, and the study population included male patients only, which might limit generalizability. The intraoperative bleeding was minimal, so we could only estimate it by visual assessment and mental computation. The current study only analyzed the safety of aspirin, clopidogrel, and warfarin in patients with IHR; the effect of relatively new oral anticoagulants remains to be analyzed. Although the European Hernia Society (EHS) guidelines have recommended the open Lichtenstein and endoscopic approach of hernia repair as the best evidence-based methods [31], in the current study, we adopted the standard UHS technique for hernia repair because all surgeries were performed by the experienced hernia surgeon who has $10+$ years' experience of the UHS technique. We consider that the reinforcement of the floor of the inguinal canal using a bilayer UHS mesh protecting the entirety of the myopectineal orifice in the preperitoneal space led to the satisfactory postoperative outcome. The rate of recurrence following the UHS technique of hernia repair in our center is minimal. Our study only focused on the safety of the UHS technique under LA. Thus, the safety and feasibility of other open mesh and non-mesh techniques, and endoscopic techniques of hernia repair with a perioperative continuation of AT, are not determined.

More research and invention of compatible and efficient AT drugs and specialization in hernia management will overall standardize healthcare in this field and provide the framework for future research. Further randomized trials are still needed to determine the best approach to the optimization of AT in this setting.

\section{Conclusions}

The present study revealed that, in the elderly, undergoing IHR with a continuation of AT did not increase the risk of intra- and postoperative hemorrhagic complications. In expert hands, the incidences of postoperative 
hemorrhagic and nonhemorrhagic complications were minimal and not severe. The few complications, if any, could be managed with relatively basic conservative measures. Thus, IHR under LA seems to be safe and feasible in the elderly with a perioperative continuation of AT.

\section{Statement of Ethics}

This study was approved by the Ethics Committees of Sichuan University, Chengdu, China (Code: 2018-482). Patients were informed regarding the character of the study, which consisted of a perioperative continuation of AT in the antithrombotic group. Relatively low risk of perioperative bleeding complications related to this protocol was clearly explained and consent was taken. All procedures performed in studies involving human participants were in accordance with the ethical standards of the institutional and/or national research committee and with the 1964 Helsinki Declaration and its later amendments or comparable ethical standards.

\section{Conflict of Interest Statement}

All authors declare no conflicts of interest.

\section{Funding Sources}

This research did not receive any specific grant from funding agencies in the public, commercial, or not-for-profit sectors.

\section{Author Contributions}

W.L. and Y.S. were involved in the conception, design, and supervision of the study. G.H. and S.Z. performed the research and data collection. Y.X., M.J., and F.J. were in charge of the acquisition, analysis, and interpretation of data for the work. G.H. wrote the first draft of the manuscript and critically revised the article for important intellectual content. All authors read and approved the final manuscript for submission.

\section{References}

1 de Goede B, Timmermans L, van Kempen BJ, van Rooij FJ, Kazemier G, Lange JF, et al. Risk factors for inguinal hernia in middle-aged and elderly men: results from the Rotterdam Study. Surgery. 2015 Mar;157(3):540-6.

2 Vandvik PO, Lincoff AM, Gore JM, Gutterman DD, Sonnenberg FA, Alonso-Coello P, et al. Primary and secondary prevention of cardiovascular disease: Antithrombotic Therapy and Prevention of Thrombosis, 9th ed: American College of Chest Physicians EvidenceBased Clinical Practice Guidelines. Chest. 2012;141(2 Suppl):e637S-8S.

3 Moscucci M, Fox KA, Cannon CP, Klein W, López-Sendón J, Montalescot G, et al. Predictors of major bleeding in acute coronary syndromes: the Global Registry of Acute Coronary Events (GRACE). Eur Heart J. 2003 Oct; 24(20):1815-23.

4 Schulman S, Beyth RJ, Kearon C, Levine MN. Hemorrhagic complications of anticoagulant and thrombolytic treatment: American College of Chest Physicians Evidence-Based Clinical Practice Guidelines (8th Edition). Chest. 2008;133(6 Suppl):257s-98s.

5 Barash P, Akhtar S. Coronary stents: factors contributing to perioperative major adverse cardiovascular events. Br J Anaesth. 2010;105 Suppl 1:i3-15.

6 Wagner ML, Khoury JC, Alwell K, Rademacher E, Woo D, Flaherty ML, et al. Withdrawal of Antithrombotic Agents and the Risk of Stroke. J Stroke Cerebrovasc Dis. 2016 Apr;25(4):902-6.

7 Verdoia M, Pergolini P, Rolla R, Nardin M, Schaffer A, Barbieri L, et al. Advanced age and high-residual platelet reactivity in patients receiving dual antiplatelet therapy with clopidogrel or ticagrelor. J Thromb Haemost. 2016 Jan;14(1):57-64.

8 Hornor MA, Duane TM, Ehlers AP, Jensen EH, Brown PS Jr, Pohl D, et al. American College of Surgeons' Guidelines for the Perioperative Management of Antithrombotic
Medication. J Am Coll Surg. 2018;227(5): 521-36.e1.

9 Banerjee S, Angiolillo DJ, Boden WE, Murphy JG, Khalili H, Hasan AA, et al. Use of Antiplatelet Therapy/DAPT for Post-PCI Patients Undergoing Noncardiac Surgery. J Am Coll Cardiol. 2017 Apr;69(14):1861-70.

10 Veitch AM, Vanbiervliet G, Gershlick AH, Boustiere C, Baglin TP, Smith LA, et al. Endoscopy in patients on antiplatelet or anticoagulant therapy, including direct oral anticoagulants: British Society of Gastroenterology (BSG) and European Society of Gastrointestinal Endoscopy (ESGE) guidelines. Gut. 2016 Mar;65(3):374-89.

11 Douketis JD, Spyropoulos AC, Spencer FA, Mayr M, Jaffer AK, Eckman MH, et al. Perioperative management of antithrombotic therapy: Antithrombotic Therapy and Prevention of Thrombosis, 9th ed: American College of Chest Physicians Evidence-Based Clinical Practice Guidelines. Chest. 2012; 141(2 Suppl):e326S-e50S.

12 Armstrong MJ, Gronseth G, Anderson DC, Biller J, Cucchiara B, Dafer R, et al. Summary of evidence-based guideline: periprocedural management of antithrombotic medications in patients with ischemic cerebrovascular disease: report of the Guideline Development Subcommittee of the American Academy of Neurology. Neurology. 2013 May;80(22): 2065-9.

13 Dindo D, Demartines N, Clavien PA. Classification of surgical complications: a new proposal with evaluation in a cohort of 6336 patients and results of a survey. Ann Surg. 2004 Aug;240(2):205-13.

14 Zhao H, Zhou L, Li L, Coon V J, Chatterton RT, Brooks DC, et al. Shift from androgen to estrogen action causes abdominal muscle fibrosis, atrophy, and inguinal hernia in a transgenic male mouse model. Proc Natl Acad Sci USA. 2018 Oct;115(44):E1042736.
15 Capodanno D, Angiolillo DJ. Antithrombotic therapy in the elderly. J Am Coll Cardiol. 2010 Nov;56(21):1683-92.

16 Lundström K-J, Sandblom G, Smedberg S, Nordin P. Risk Factors for Complications in Groin Hernia Surgery: A National Register Study. Ann Surg. 2012 Apr;255(4):784-8.

17 Köckerling F, Roessing C, Adolf D, SchugPass C, Jacob D. Has endoscopic (TEP, TAPP) or open inguinal hernia repair a higher risk of bleeding in patients with coagulopathy or antithrombotic therapy? Data from the Herniamed Registry. Surg Endosc. 2016 May;30(5): 2073-81.

18 Broderick JP, Bonomo JB, Kissela BM, Khoury JC, Moomaw CJ, Alwell K, et al. Withdrawal of antithrombotic agents and its impact on ischemic stroke occurrence. Stroke. 2011 Sep;42(9):2509-14.

19 Garcia DA, Regan S, Henault LE, Upadhyay A, Baker J, Othman M, et al. Risk of thromboembolism with short-term interruption of warfarin therapy. Arch Intern Med. 2008 Jan; 168(1):63-9.

20 Akopov SE, Suzuki S, Fredieu A, Kidwell CS, Saver JL, Cohen SN. Withdrawal of warfarin prior to a surgical procedure: time to follow the guidelines? Cerebrovasc Dis. 2005;19(5): 337-42.

21 Collet JP, Montalescot G, Blanchet B, Tanguy ML, Golmard JL, Choussat R, et al. Impact of prior use or recent withdrawal of oral antiplatelet agents on acute coronary syndromes. Circulation. 2004 Oct;110(16):2361-7.

22 Beving $\mathrm{H}$, Zhao C, Albage A, Ivert T. Abnormally high platelet activity after discontinuation of acetylsalicylic acid treatment. Blood Coagul Fibrinolysis. 1996 Jan;7(1):80-4.

23 Sacco RL, Diener HC, Yusuf S, Cotton D, Ounpuu S, Lawton WA, et al.; PRoFESS Study Group. Aspirin and extended-release dipyridamole versus clopidogrel for recurrent stroke. N Engl J Med. 2008 Sep;359(12):123851. 
24 Ulrych J, Kvasnicka T, Fryba V, Komarc M, Malikova I, Burget F, et al. 28 day post-operative persisted hypercoagulability after surgery for benign diseases: a prospective cohort study. BMC Surg. 2016 Apr;16(1):16.

25 Ong W, Shen T, Tan WB, Lomanto D. Is preoperative withdrawal of aspirin necessary in patients undergoing elective inguinal hernia repair? Surg Endosc. 2016 Dec;30(12):5542-9.

26 Lee LD, Hausen G, Aschenbrenner K, Stroux A, Kreis ME, Lauscher JC. Perioperative Platelet Inhibition in Elective Inguinal Hernia Surgery-Increased Rate of Postoperative
Bleeding and Hematomas? Int Surg. 2019; 103(1-2):40-7.

27 Smoot RL, Oderich GS, Taner CB, Greenlee SM, Larson DR, Cragun EB, et al. Postoperative hematoma following inguinal herniorrhaphy: patient characteristics leading to increased risk. Hernia. 2008 Jun;12(3):261-5.

28 Sanjay P, Woodward A. Inguinal hernia repair: local or general anaesthesia? Ann R Coll Surg Engl. 2007 Jul;89(5):497-503.

29 Hayse-Gregson PB, Achola KJ, Smith G. Changes in haemodynamics and plasma catecholamine concentrations after field block for inguinal herniorrhaphy using lignocaine with adrenaline. Anaesthesia. 1990 Jan;45(1): $7-10$

30 Issıl RG, Yazıcı P, Demir U, Kaya C, Bostancı Ö, İdiz UO, et al. Approach to inguinal hernia in high-risk geriatric patients: should it be elective or emergent? Ulus Travma Acil Cerrahi Derg. 2017 Mar;23(2):122-7.

31 Simons MP, Aufenacker T, Bay-Nielsen M, Bouillot JL, Campanelli G, Conze J, et al. European Hernia Society guidelines on the treatment of inguinal hernia in adult patients. Hernia. 2009 Aug;13(4):343-403. 